Case Report

\title{
Costello Syndrome and Umbilical Ligament Rhabdomyosarcoma in Two Pediatric Patients: Case Reports and Review of the Literature
}

\author{
Carlos Sánchez-Montenegro, ${ }^{1,2}$ Alejandra Vilanova-Sánchez, ${ }^{2}$ Saturnino Barrena-Delfa, ${ }^{2}$ \\ Jair Tenorio, ${ }^{3,4}$ Fernando Santos-Simarro, ${ }^{3,4}$ Sixto García-Miñaur, ${ }^{3,4}$ Pablo Lapunzina, ${ }^{3,4}$ \\ and Leopoldo Martínez-Martínez ${ }^{2}$ \\ ${ }^{1}$ Pediatric Surgical Oncology Section, Oncology Service, Department of Hematology-Oncology, \\ Hospital Nacional de Niños "Dr. Carlos Sáenz Herrera”, Paseo Colón, 1654-1000 San José, Costa Rica \\ ${ }^{2}$ Oncology Section, Department of Pediatric Surgery, Hospital Universitario La Paz, Paseo de la Castellana 261, 28046 Madrid, Spain \\ ${ }^{3}$ Institute of Medical and Molecular Genetics (INGEMM), Instituto de Investigación Hospital Universitario La Paz (IdiPAZ), \\ Paseo de la Castellana 261, 28046 Madrid, Spain \\ ${ }^{4}$ Centro de Investigación Biomédica en Red de Enfermedades Raras (CIBERER), Instituto de Salud Carlos III (ISCIII), \\ Calle Sinesio Delgado 4, 28029 Madrid, Spain \\ Correspondence should be addressed to Carlos Sánchez-Montenegro; csanchezm@salud.madrid.org
}

Received 5 October 2016; Revised 20 December 2016; Accepted 28 December 2016; Published 19 January 2017

Academic Editor: Philip D. Cotter

Copyright (C) 2017 Carlos Sánchez-Montenegro et al. This is an open access article distributed under the Creative Commons Attribution License, which permits unrestricted use, distribution, and reproduction in any medium, provided the original work is properly cited.

\begin{abstract}
Costello syndrome is caused by heterozygous de novo missense mutations in the protooncogene HRAS with tumor predisposition, especially rhabdomyosarcoma. We here report two pediatric patients with Costello syndrome and umbilical ligament rhabdomyosarcoma. A review of the literature published in English in MEDLINE from January 1971 to June 2016 using the search terms "Costello syndrome" and "rhabdomyosarcoma" was performed, including two new cases that we describe. Twenty-six patients with Costello syndrome and rhabdomyosarcoma were recorded with mean age of diagnosis of 2 years and 8 months. The most common tumor location was the abdomen/pelvis, including four out of ten of those in the umbilical ligament. The most common histological subtype was embryonal rhabdomyosarcoma. Overall survival was $43 \%$. A total of 17 rhabdomyosarcomas in pediatric patients arising in the umbilical ligament were recorded with mean age of diagnosis of 3 years and 4 months. Overall survival was $69 \%$. Costello syndrome is a poorly known disorder in pediatric oncology but their predisposition to malignancies implies the need for a new perspective on early diagnosis and aggressive medical and surgical treatment.
\end{abstract}

\section{Introduction}

Costello syndrome (CS) (OMIM \#218040) is a rare disorder with a distinctive prenatal presentation, postnatal feeding difficulties and failure to thrive, characteristic facial appearance (coarse features, full lips, and large mouth), abnormalities of the heart (hypertrophic cardiomyopathy, pulmonary valve stenosis, and tachyarrhythmia), skin and musculoskeletal system (soft skin, deep palmar and plantar creases, papillomata, sparse or curly hair, joint laxity, ulnar deviation of wrist and fingers, and tight Achilles tendons), and tumor predisposition such as rhabdomyosarcoma (RMS), neuroblastoma, or transitional cell carcinoma of the bladder [1]. It was first described in 1971 by Costello, a New Zealand pediatrician, based on its distinctive phenotype [2-4]. CS is caused by heterozygous de novo missense mutations in the protooncogene Harvey rat sarcoma viral oncogene homolog (HRAS) (chromosome 11p15.5). These mutations are present in $100 \%$ of patients with CS, but they are usually confirmed in $80-90 \%$ due to an inaccurate clinical diagnosis $[4,5]$. Mutations result in a gain-of-function of the abnormal protein product and increased activation of the Mitogen 


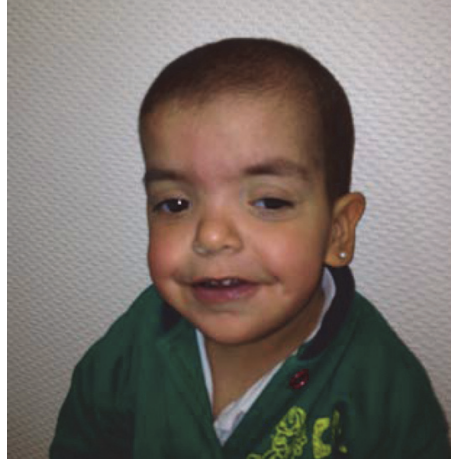

(a)

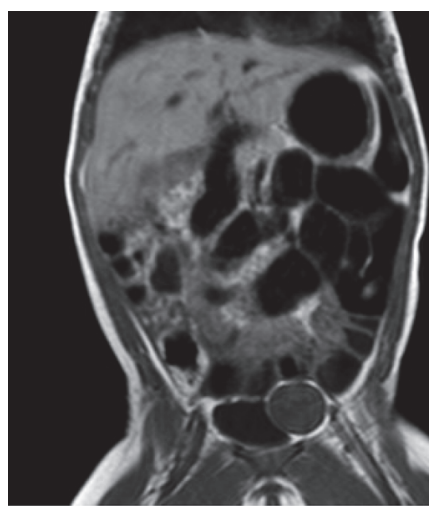

(d)

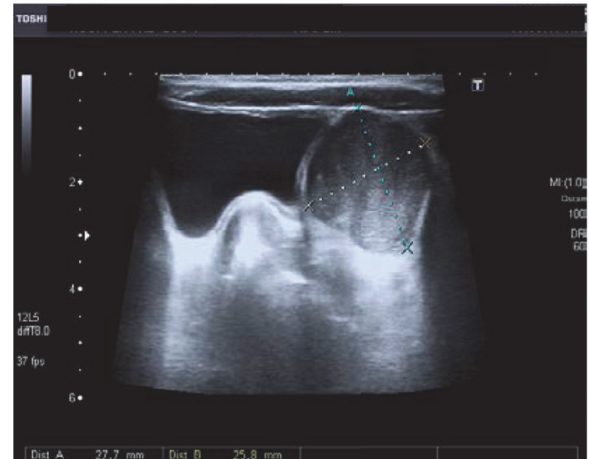

(b)

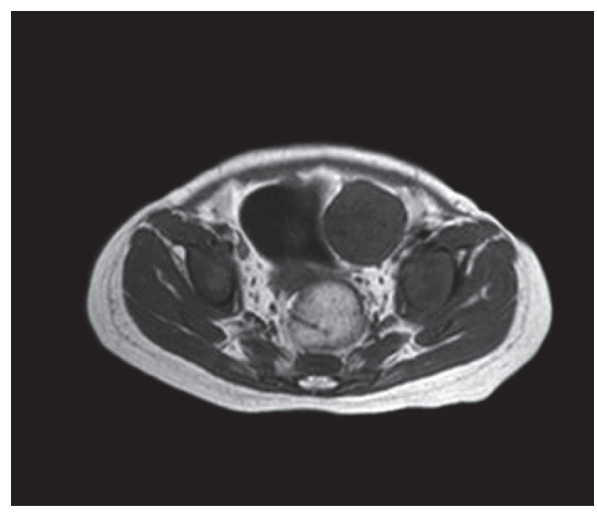

(e)

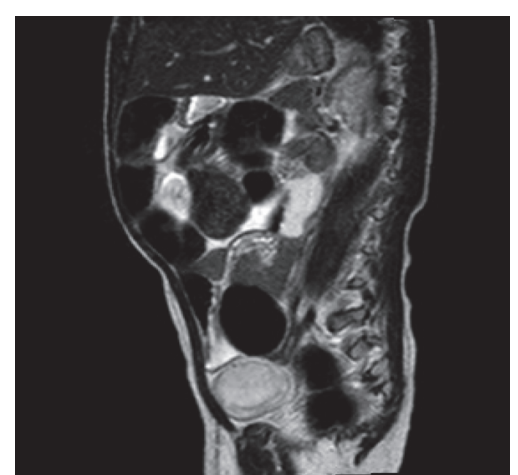

(c)

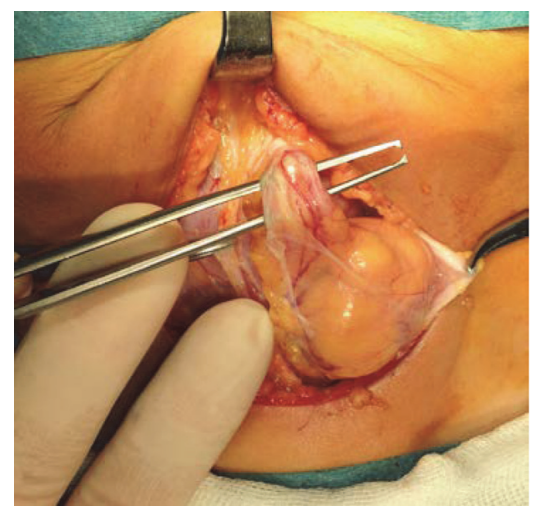

(f)

Figure 1: Patient 1. (a) Photograph of the physical features of the patient with low-set ears, lower palpebral fissures, hypertelorism, broad nasal bridge, thick lips, and thin hair. (b) Abdominal ultrasound showing the presence of a solid mass paravesical displacing the left ovary and bladder without infiltrate surrounding structures. (c) Initial MRI showing a hyperintense tumor predominantly heterogeneous intensity on T2-weighted sequence compressing the bladder. ((d) and (e)) MRI on T1-weighted sequences after 3 cycles of chemotherapy with slight decrease in left paravesical oval mass. (f) Operative image indicating the presence of tumor originating in the left medial UL.

activated protein kinase (MAPK) pathway [1]. The most common tumor associated with CS is RMS, mainly involving abdomen, pelvis, and/or urogenital area [6]. The first two CS patients associated with RMS were described in 1998 [7]; before that date this syndrome was mainly associated with benign tumors, especially papillomata [3].

We here report two children with CS and RMS of the umbilical ligament (UL). No prior correlation of this tumor location with CS had been described so far.

\section{Case Presentation}

2.1. Case 1. This is a 4.4-year-old female. She is third child of healthy unrelated parents with no family history of note. Pregnancy was complicated with polyhydramnios. The patient was born from a preterm delivery (33 weeks), large (2840 gr) for gestational age. She presented feeding difficulties from the beginning that required nasogastric tube feeding and later gastrostomy, at the age of 4 months. Cardiological examination showed mild pulmonary stenosis. She was referred to the Genetics Clinic for assessment at the age of 6 months because of failure to thrive, hypotonia, and dysmorphic features. On examination she had features highly suggestive of a RASopathy, but on account of the skin findings (sparse hair, glabellar capillary malformation, a large $4 \times 12 \mathrm{~cm}$ café-au-lait spot in her right flank, and two blue nevi in right temporal region and left groin), difficult to differentiate with certainty between CS and Cardiofaciocutaneous syndrome. However, HRAS mutation analysis demonstrated a common recurrent mutation (p.G12S), confirming the diagnosis of CS in this child at the age of 7 months (Figure 1(a)). Because of the high risk of tumor development associated with this condition, close follow-up was indicated according to the recommended tumor screening protocol [8, 20]. An abdominal ultrasound scan performed at the age of 16 months revealed a $31 \times 32 \mathrm{~mm}$ paravesical solid pelvic mass, with minimal intralesional vascularity; no intraabdominal free fluid or lymphadenopathies were seen. The ovaries were not involved (Figure 1(b)). Magnetic resonance imaging $(\mathrm{MRI})$ showed a left paravesical tumor $(3 \times 5 \times 2.7 \mathrm{~cm})$ without associated lymphadenopathies, hypointense on T1weighted sequences, and hyperintense with predominantly heterogeneous intensity on T2-weighted sequences. The mass caused compression and displacement of the bladder to the right side (Figure 1(c)). Tumor markers were as follows: beta-subunit of human chorionic gonadotropin $(\beta$ hCG $)<2 \mathrm{mUI} / \mathrm{mL}$ (normal $<6.0$ ), alpha-fetoprotein $(\alpha \mathrm{FP})$ $2.9 \mathrm{ng} / \mathrm{mL}$ (normal $<8.0$ ), carcinoembryonic antigen $(\mathrm{CEA})$ 
$0.8 \mathrm{ng} / \mathrm{mL}$ (normal < 5.0), CA-15.3 19.7 UI/mL (normal < 32.0), CA-19.9 43.7 UI/mL (normal < 37.0), and neuron specific enolase (NSE) $27.86 \mathrm{ng} / \mathrm{mL}$ (normal 0-16.0). Thoracic computed tomography (CT) and scintigraphy showed no evidence of metastasis. Bone marrow biopsy was normal. Tru-Cut biopsy guided by ultrasound reported an embryonal RMS with a proliferation index Ki-67 of $60 \%$, mesenchymal malignant round cell, and short spindle tumor: vimentin $(+)$, desmin (focal + ), MyoD1 (focal + ), CD99 (+ focal staining), S100 protein (-), pancytokeratins AE1/AE3 (-), synaptophysin (-), and CD99 (-). She was enrolled on the standard risk group protocol European pediatric Soft tissue Sarcoma Study Group (EpSSG) RMS 2005 subgroup D with 3 neoadjuvant chemotherapy cycles. Preoperative MRI showed a slight decrease in tumor size $(3.2 \times 3 \times 2.5 \mathrm{cms})$ (Figures 1(d) and 1(e)). At the definitive surgery a $5 \mathrm{~cm}$ tumor dependent of the remaining left umbilical artery (left medial UL), located slightly to the left and displacing the bladder dome, was found (Figure 1(f)). Complete resection of the tumor was performed. No pathological lymph nodes were observed. Final pathology report showed an embryonal RMS with signs of response to chemotherapy (maturation phenomenon at cytoplasmic and nuclear level of the tumor cellularity), with maximum cell proliferation index Ki-67 of $5-10 \%$, almost all undifferentiated except in a small region; resection margins were negative for tumor $(1-4 \mathrm{~mm}$ from the edges of radial resection margin and $3 \mathrm{~cm}$ of $\mathrm{UL}$ ). Chemotherapy was then continued until completing 25 weeks and coadjuvant radiotherapy was administrated after the completion of chemotherapy. At 23 months of follow-up after diagnosis, no residual tumor or relapse has been identified.

2.2. Case 2. This is a 5.4-year-old female. She is the first and only child of a healthy nonconsanguineous couple with unremarkable family history. Pregnancy was complicated with polyhydramnios and delivery was preterm (34 weeks), with a high birth weight $(2980 \mathrm{~g},+2.6 \mathrm{SD})$. She was admitted to the neonatal ward for investigation of a suspected dysmorphic syndrome and initial FGFR3 molecular genetic testing for achondroplasia revealed no mutations. Feeding difficulties and failure to thrive were noted from the beginning requiring nutritional intervention and gastrostomy feeding from the age of 10 months. She had a heart murmur with a normal echocardiogram. She also had bronchomalacia and at MRI nonspecific white matter hyperintensities in CNS. She was referred to our clinical Genetics Clinic for evaluation at the age of 4 months and in view of her previous history and clinical findings CS was suspected. HRAS Sanger sequencing detected the common c.34G $>$ A (p.G12S) mutation confirming the diagnosis of CS (Figure 2(a)). As part of the followup the recommended tumor screening protocol was arranged and at the age of 3 years she underwent an abdominal ultrasound that showed a tubular image running through the theoretical place of the umbilical arteries, with a larger caliber on the right side of $5.6 \mathrm{~mm}$, and associated echoes inside without Doppler flow (Figure 2(b)). Two subsequent ultrasounds did not show any abnormality. A new abdominal ultrasound 9 months later reported the presence of a hypoechoic multilobulated solid tumor $(5.3 \times 2.8 \mathrm{~cm})$ in the midline extending to the right in supravesical region with marked hypervascularization and extending cranially to the vicinity of the abdominal wall (Figures 2(c) and 2(d)). MRI showed a solid abdominal tumor $(6 \times 5.5 \times 3 \mathrm{~cm})$ composed of nodular masses grouped in the UL region with involvement of the medial umbilical folds that were thickened throughout the iliac vessels region (Figure 2(e)). No regional metastatic disease was observed. Tumor markers were as follows: $\alpha \mathrm{FP}$ $<1.3 \mathrm{ng} / \mathrm{mL}$, CEA $5.3 \mathrm{ng} / \mathrm{mL}$, and CA-19.9 UI/mL. No lung metastases were observed in the thoracic CT scan and bone marrow biopsy was normal. She underwent a complete tumor resection (Figure 2(f)). The mass was located on the right medial UL (from its insertion into the right iliac artery to the umbilicus). Pathology report showed a spindle cell RMS with microscopic positive margins in 2 submitted samples $(8.5 \times 5 \times 3 \mathrm{~cm}$ and $3.5 \times 1.5 \times 1 \mathrm{~cm})$ and one resected lymph node was negative for malignancy. Tumor was composed of spindle cells that form cross linked bundles varying degrees, with elongated oval nuclei, vesicular configurations with coarse chromatin. The tumor had a pattern of nodular growth, markedly myxoid stroma with microcystic focal degeneration and 5\% tumor necrosis; no lymphovascular invasion, maximum proliferation index $\mathrm{Ki} 67$ of 30\%; muscle specific actin (+), desmin (+), WT1 (+ cytoplasmic), MyoD1 (+ nuclear), and CD99 (-). Two weeks after surgery, the standard risk group protocol EpSSG RMS 2005 subgroup B (Stage I) chemotherapy was started that includes ifosfamide, vincristine, and actinomycin D. At 18 months of followup after diagnosis, no residual tumor or relapse has been identified.

\section{Methods}

We describe two new cases of RMS and CS, based on medical records of patients diagnosed at the Institute of Medical and Molecular Genetics and treated at the Departments of Pediatric Hematology-Oncology and Pediatric Surgery, University Hospital La Paz (Madrid, Spain). A review of the literature published in English in MEDLINE from January 1971 to June 2016 using the search terms CS and RMS was done; also the references contained in these articles even if they were not indexed in MEDLINE were analyzed. A review from January 1933 until June 2016 of all reported pediatric cases of UL RMS, including as search criteria UL, RMS, urachus, and umbilical arteries, was performed.

\section{Results}

In all cases analyzed and reviewed (same cases reported in more than one publication were counted once), it was possible to document the presence of 26 patients with CS and RMS (Table 1) [5-25], including the 2 cases described herein. The mean age of diagnosis was 2 years and 8 months (range: 6 months-7 years). It was slightly more common in females than in males $(1.2: 1)$. The commonest location was the abdomen/pelvis (10/20), including 4 out of 10 of those in the UL. The most common histological type was embryonal RMS (14/19). Seven out of nine patients who died (and with reported histology) showed embryonal RMS. The 


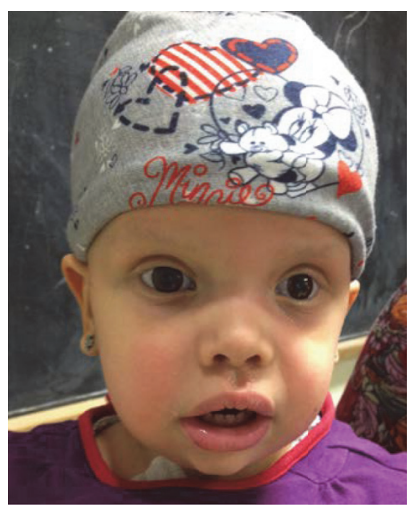

(a)

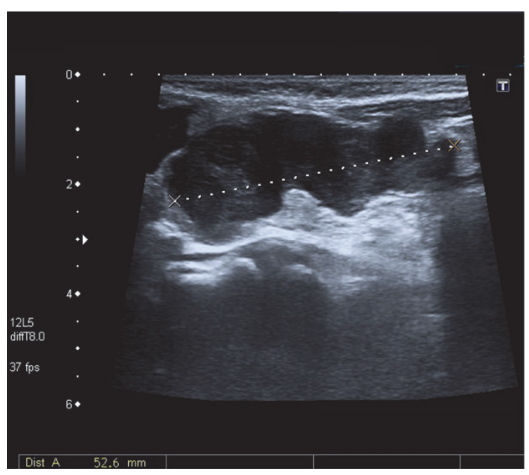

(d)

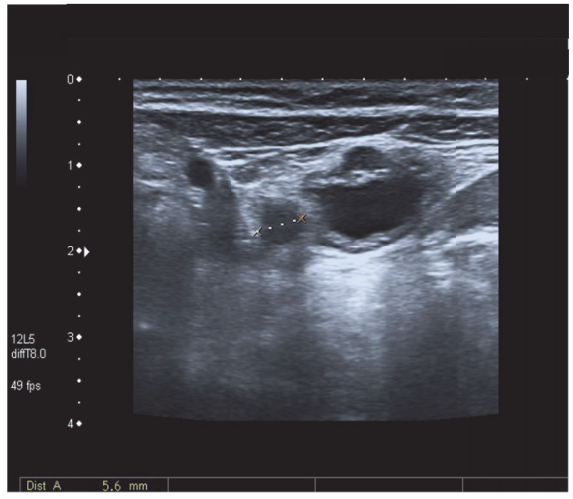

(b)

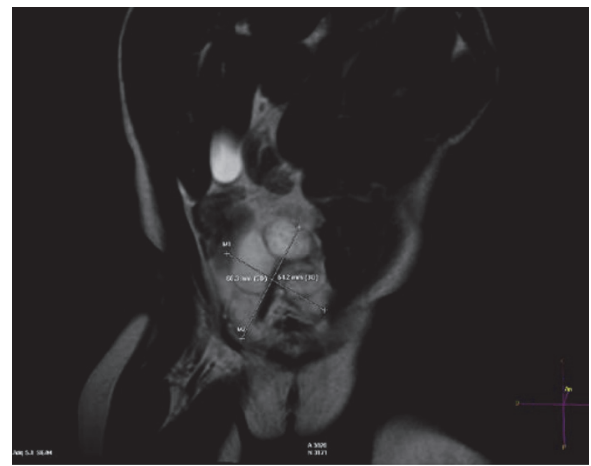

(e)

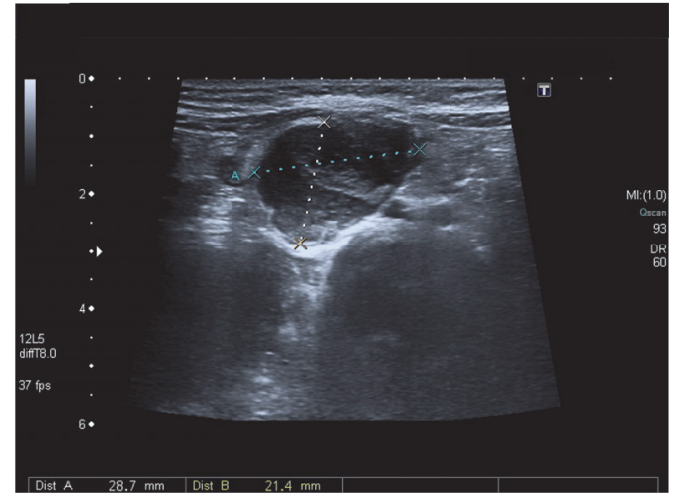

(c)

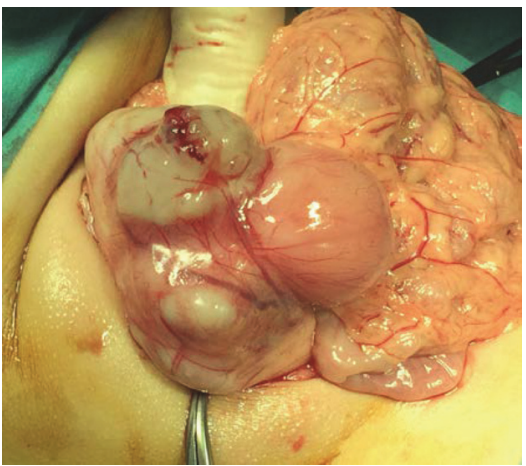

(f)

Figure 2: Patient 2. (a) Photograph of the physical features of the patient with prominent forehead, low-set ears, lower palpebral fissures, hypertelorism, broad nasal bridge, thick lips, and anteverted slightly nostrils. (b) Initial abdominal ultrasound showing the presence of a tubular image caliber of $5.6 \mathrm{~mm}$ extending in the theoretical location of the right umbilical artery. ((c) and (d)) Follow-up abdominal ultrasound in the midline showing a multilobulated hypoechoic solid tumor with marked hypervascularization, extending from the proximity of the abdominal wall to the right supravesical region. (e) MRI onT2-weighted sequence confirms the presence of several nodular masses grouped with thickening of both medial umbilical folds, the right to $4 \mathrm{~mm}$ proximal to the umbilicus and $8 \mathrm{~mm}$ adjacent to the iliac vessels paravesical region portion. (f) Operative image indicating the presence of tumor originating in the right medial UL.

most frequent encountered HRAS mutation associated with RMS was p.G12S (7/12). Only 4 deaths were documented in patients with change in codon 12 (2 patients G12S, 1 patient G12A, and 1 patient G12C). Chemotherapy that was applied varies based on the protocols of the countries where they were diagnosed (Table 2) [5-10, 12-14, 16-25]. Overall survival was about $43 \%(9 / 21)$. Mortality was observed in 12 out of 21 individuals; in nine of them associated with neoplasia; and in three associated with surgery.

Similarly, the total number of reported cases of UL RMS in pediatric patients, either in the obliterated umbilical arteries (medial UL) or in urachus (median UL), is 17 including these 2 new cases (Table 3) [5, 7-9, 24-31]. The mean age of diagnosis was 3 years and 4 months (range: 4 months -6 years). It is slightly more common in females than in males $(1.4: 1)$. The most common location was the urachus (15/17). The most common histological type was embryonal RMS (12/15) and one-fourth (3/12) of patients who died with reported histology showed embryonal RMS. Chemotherapy varies based on the protocols of the countries where they were diagnosed (Table 4) [7, 24, 26-31]. Overall survival was 69\%
(11/16), overall mortality was $31 \%(5 / 16)$, two-year absolute survival rate was $69 \%(11 / 16)$, and five-year absolute survival rate was $19 \%(3 / 16)$.

\section{Discussion}

The prevalence of CS is estimated to be $1: 1,290,000$ individuals in Japan and at least 1:500,000 in the United Kingdom; the incidence was estimated to be 1:60,000-100,000 [4, 22]. The overall tumor incidence is approximately $10-15 \%$ over the lifetime of individuals with an identified HRAS mutation $[1,4,9]$, RMS accounting in about $60 \%$ of all neoplasia [1]. Based on the 300 cases of CS currently known [4], we found an incidence of $8.7 \%$ RMS in patients with CS.

CS is the first disorder associated with germline mutations in the Ras family of guanosine- $5^{\prime}$-triphosphate (GTP)ases [21]. HRAS has six exons; five exons are encoded for a protein of 189 amino acids with a molecular weight of $21 \mathrm{kDa}$ [4]. Missense mutations at codons 12 and 13 are in constitutively active GTP-bound conformation and activate downstream effectors such as MAPK, phosphatidylinositol 3-kinases (PI3K), and ral guanine nucleotide dissociation 


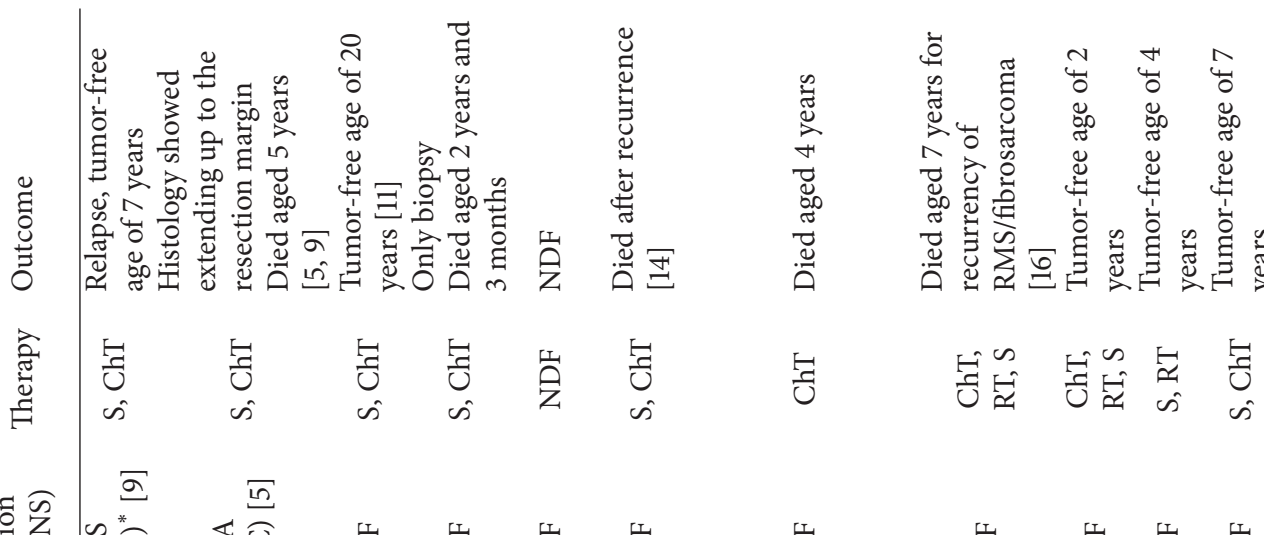

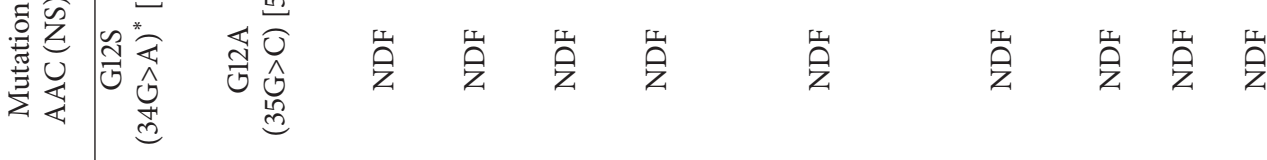

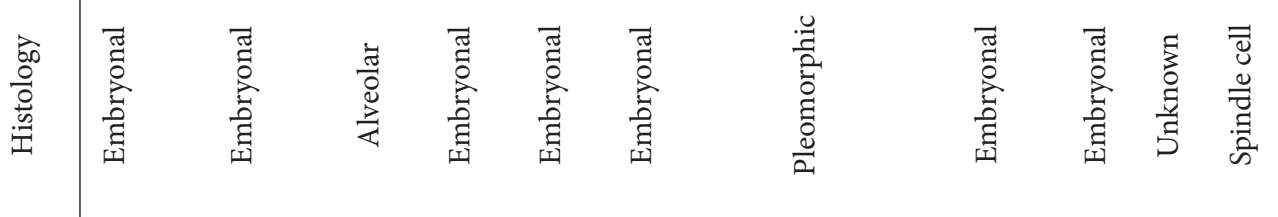

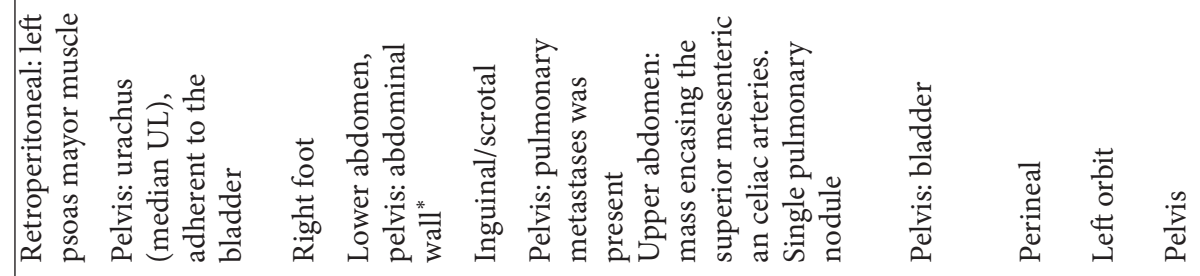

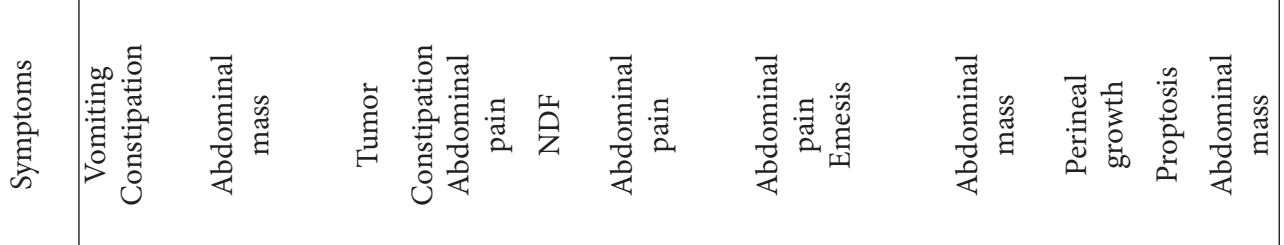

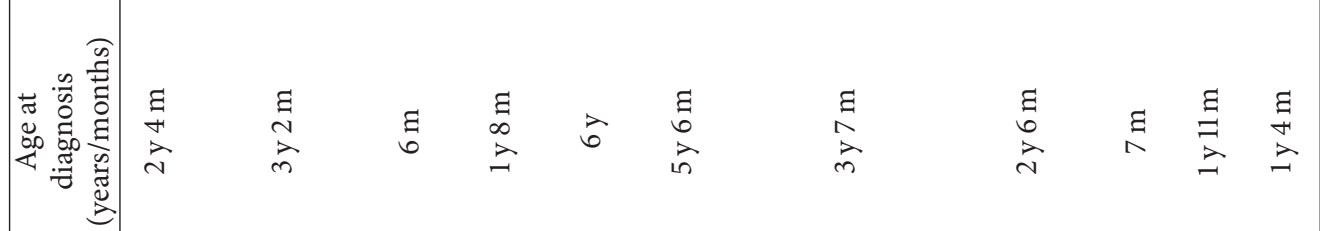

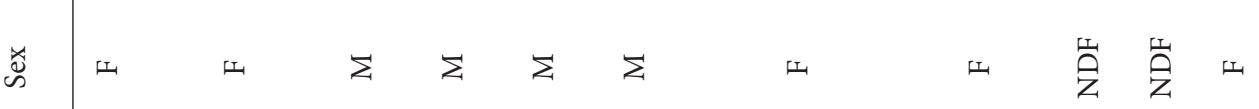

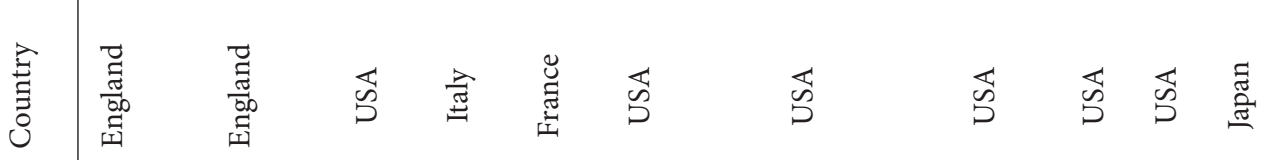

ॠ

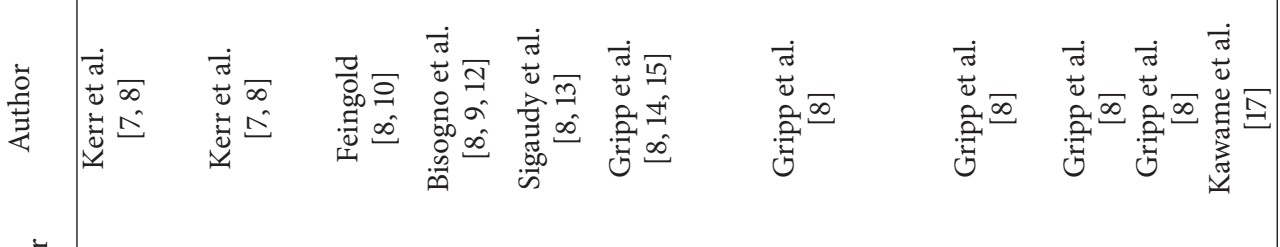

芯言 


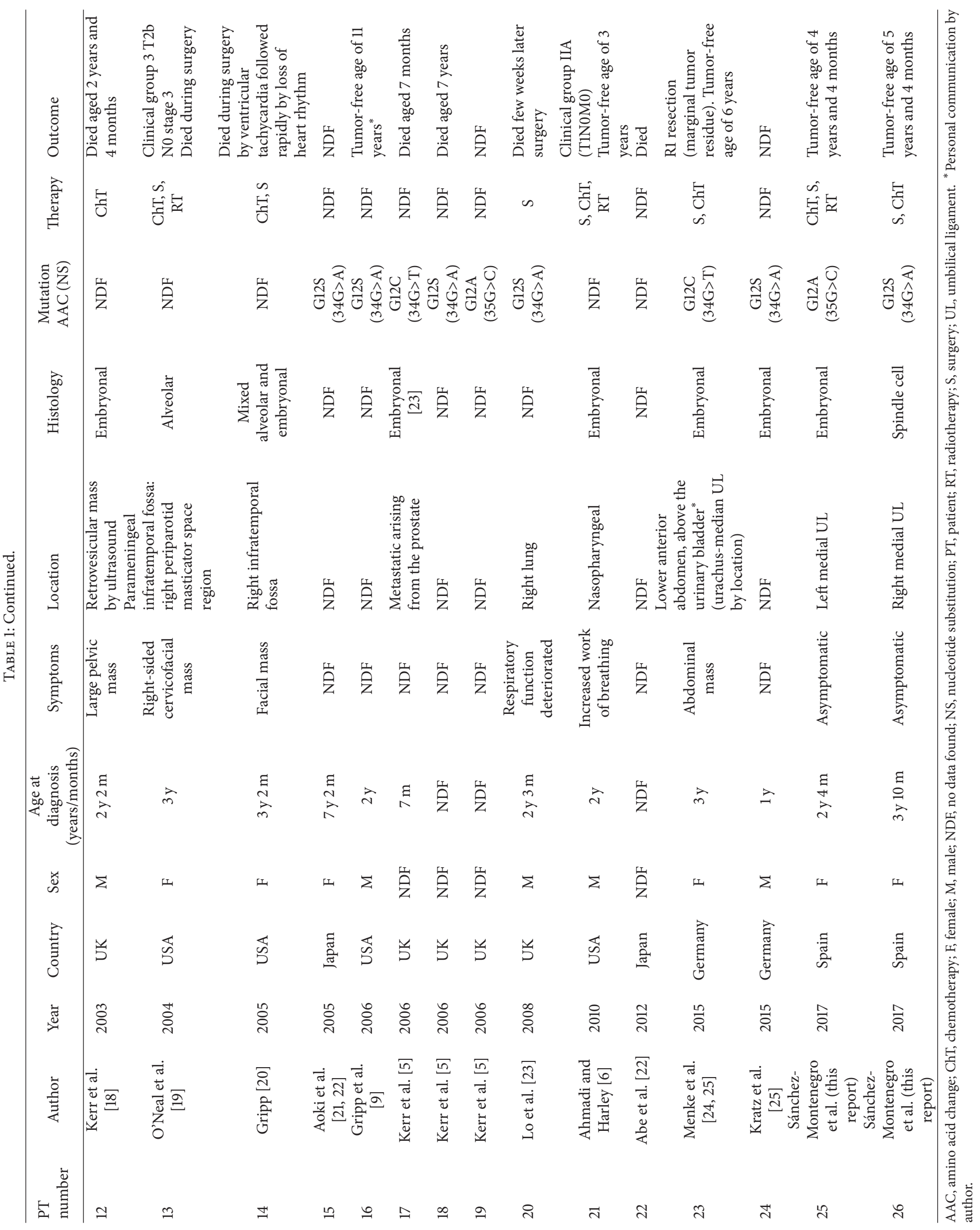


TABLE 2: Chemotherapy of patients with Costello syndrome and rhabdomyosarcoma.

\begin{tabular}{|c|c|}
\hline $\begin{array}{l}\text { PT } \\
\text { number }\end{array}$ & Chemotherapy protocol \\
\hline 1 & $\begin{array}{l}\text { Initially with VCR, ACD, and CTX. After debulking continued on ChT using IFOS, VCR, and ACD. After the second course, } \\
\text { when she developed IFOS induced encephalopathy, she was changed back to pulses of VCR, ACD, and CTX given every } 3 \\
\text { weeks for } 9 \text { courses [7] }\end{array}$ \\
\hline 2 & $\begin{array}{l}\text { Initially IFOS, ACD, and VCR. After } 4 \text { courses, CTX was substituted for IFOS because hemorrhagic cystitis. Tx complicated } \\
\text { by recurrent episodes of moderately severe diarrhoea and febrile neutropenia requiring readmission to hospital. Tx was } \\
\text { completed after six courses of ChT [7] }\end{array}$ \\
\hline 3 & Tx consisted of below the knee amputation and ChT (DOX, ACD, VCR, and CTX) [10] \\
\hline 4 & $\begin{array}{l}\text { Tx was implemented according to the Italian protocol for pediatric soft tissue sarcoma. Three courses of ChT, including VCR } \\
(0.025 \mathrm{mg} / \mathrm{kg} \text { day } 1) \text {, IFOS }(50 \mathrm{mg} / \mathrm{kg} \text { days } 1 \text { and } 2) \text {, and alternating ACD }(0.025 \mathrm{mg} / \mathrm{kg} \text { day } 1) \text { and DOX }(0.7 \mathrm{mg} / \mathrm{kg} \text { day } 1) \text { were } \\
\text { administered. Symptoms regressed, and the CT scan showed a reduction in tumor volume of more than two-thirds. After } 3 \\
\text { more courses of ChT, the patient experienced abdominal pain. A repeat abdominal CT scan showed an increase in the pelvic } \\
\text { mass. Despite the administration of a different ChT with CBDCA ( } 11 \mathrm{mg} / \mathrm{kg} \text { day } 1) \text { and VP-16 ( } 3 \mathrm{mg} / \mathrm{kg} \text { for } 3 \text { doses), the tumor } \\
\text { continued to grow, and the child died } 7 \text { month after the diagnosis of RMS [12] }\end{array}$ \\
\hline 5 & NDF [13] \\
\hline 6 & $\begin{array}{l}\text { The first cycle of ChT with VCR, ACD, and CTX and topotecan reduced the tumor size [16]. The tumor was surgically } \\
\text { removed after the first ChT [8]. Debulking S was required because of rapid growth and tumor necrosis. The protocol was } \\
\text { continued and was deemed sufficiently successful to withhold RT. One month after completion of the first protocol, tumor } \\
\text { recurrence necessitated a second round of ChT with irinotecan and DOX. The latter drug was administered about } 1 \text { week } \\
\text { before he died, during which time he had tachycardia (heart rate } 180-20 \mathrm{bpm} \text { ) [14] }\end{array}$ \\
\hline 7 & The tumor did not respond to ChT and she died shortly after her 4th birthday [8] \\
\hline 8 & Tx was a combination of S, ChT, and RT, continued until age of 3.5 years [8] \\
\hline $9-10$ & NDF [8] \\
\hline 11 & PT had later generalized tonic-clonic seizures during her Tx for RMS [17] \\
\hline 12 & Despite extensive ChT, the tumor progressed rapidly and the PT died 2 months later [18] \\
\hline 13 & $\begin{array}{l}\text { Induction ChT was commenced with the initial 12-week course of VCR, ACD, and CTX based on the Intermediate Risk } \\
\text { Protocol D } 9803 \text { of the COG [19] }\end{array}$ \\
\hline 14 & ChT did not result in tumor shrinkage; therefore, surgical resection was performed [20] \\
\hline $15-22$ & $\operatorname{NDF}[5,6,9,21-23]$ \\
\hline 23 & ChT according to the cooperative soft tissue sarcoma protocol (CWS-2002 P) was well tolerated by the PT [24] \\
\hline 24 & NDF [25] \\
\hline 25 & $\begin{array}{l}\text { ChT in the standard risk group protocol EpSSG RMS } 2005 \text { subgroup D, } 3 \text { preoperative cycles of IFOS ( } 2 \text { doses at } 100 \mathrm{mg} / \mathrm{kg}) \text {, } \\
\text { VCR }(0.05 \mathrm{mg} / \mathrm{kg}) \text {, and ACD }(0.05 \mathrm{mg} / \mathrm{kg})\end{array}$ \\
\hline 26 & $\begin{array}{l}\text { ChT (postoperative } 15 \text { days) in the standard risk group protocol EpSSG RMS } 2005 \text { subgroup B (Stage I), cycles of IFOS (2 } \\
\text { doses of } 3 \mathrm{~g} / \mathrm{m}^{2} / \mathrm{d} \text { ), VCR }\left(1.5 \mathrm{mg} / \mathrm{m}^{2}\right) \text {, and ACD }\left(1.5 \mathrm{mg} / \mathrm{m}^{2}\right) \text {, was initiated }\end{array}$ \\
\hline
\end{tabular}

ACD, actinomycin D; CBDCA, carboplatin; ChT, chemotherapy; COG, children's oncology group; CTX, cyclophosphamide; CT, computed tomography; CWS, Cooperative Weichteilsarkom Studie (Cooperative Soft Tissue Sarcoma Study); DOX, Doxorubicin; EpSSG, European pediatric Soft tissue sarcoma Study Group; IFOS, ifosfamide; NDF, no data found; PT, patient; RMS, rhabdomyosarcoma; RT, radiotherapy; S, surgery; Tx, treatment; VCR, vincristine; VP-16, etoposide.

stimulator (RalGDS) [16, 21], in signaling pathways controlling cell proliferation and differentiation [9].

The majority of patients with CS had $34 \mathrm{G}>\mathrm{A}$ transition in codon $12(80 \%)[4,16]$. More than $95 \%$ of the mutations are predicted to result in substitution of the glycine in position 12 or 13 of the protein product [1]. All individuals with malignancy had a codon 12 mutation (especially G12A, 4/7 patients) $[5,21]$. The rare G12V mutation is associated with a more severe, early lethal phenotype; some patients die from respiratory distress, hypertrophic cardiomyopathy, or malignant tachycardia prior to being diagnosed with CS [1]. As far as we know, no individual with G13C has developed a malignant tumor $[4,32]$.
RMS is the most common soft tissue sarcoma of infancy. Its incidence is between 4 and 7 cases per 1 million children younger than 15 years $[8,12]$. The age peak is between 2 and 5 years with a slight male predominance (1.2-1.4/1). These tumors may arise anywhere in the body and fewer than $20 \%$ are located in the pelvis [12]. Most RMS tumors are originated in the head and neck region, urogenital tract, and extremities [33]. The most common histologic type seen in $>50 \%$ is embryonal, followed by alveolar in 20-30\%. Pleomorphic RMS is rare (1\%) and has a poor prognosis [8]. Alveolar RMS is characterized by the translocation $\mathrm{t}(2 ; 13)(\mathrm{q} 35 ; \mathrm{q} 14)$ in $70 \%$ of cases and the variant translocation $\mathrm{t}(1 ; 13)(\mathrm{p} 36 ; \mathrm{q} 14)$ in a smaller percentage of cases. Evidence accumulates 


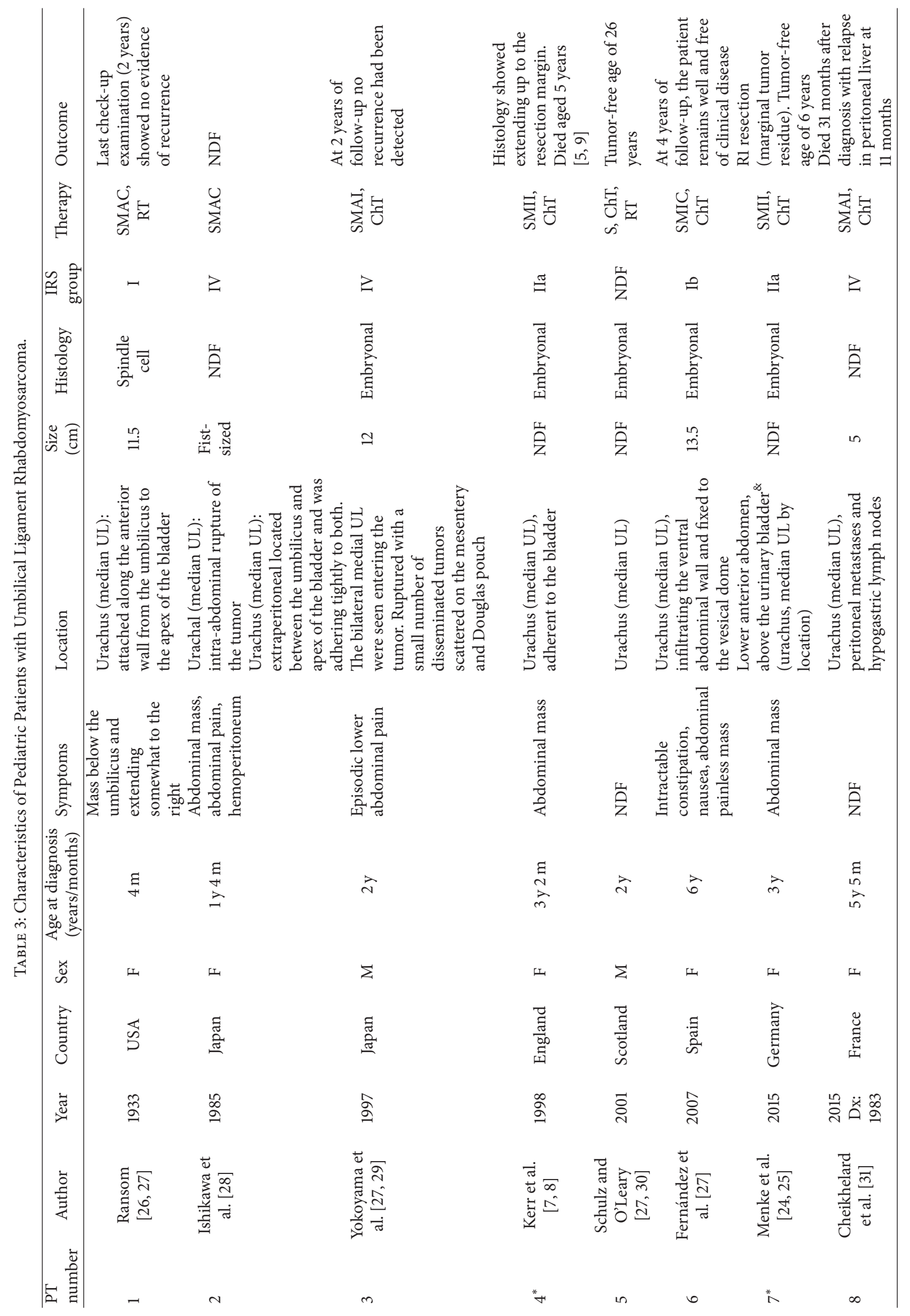




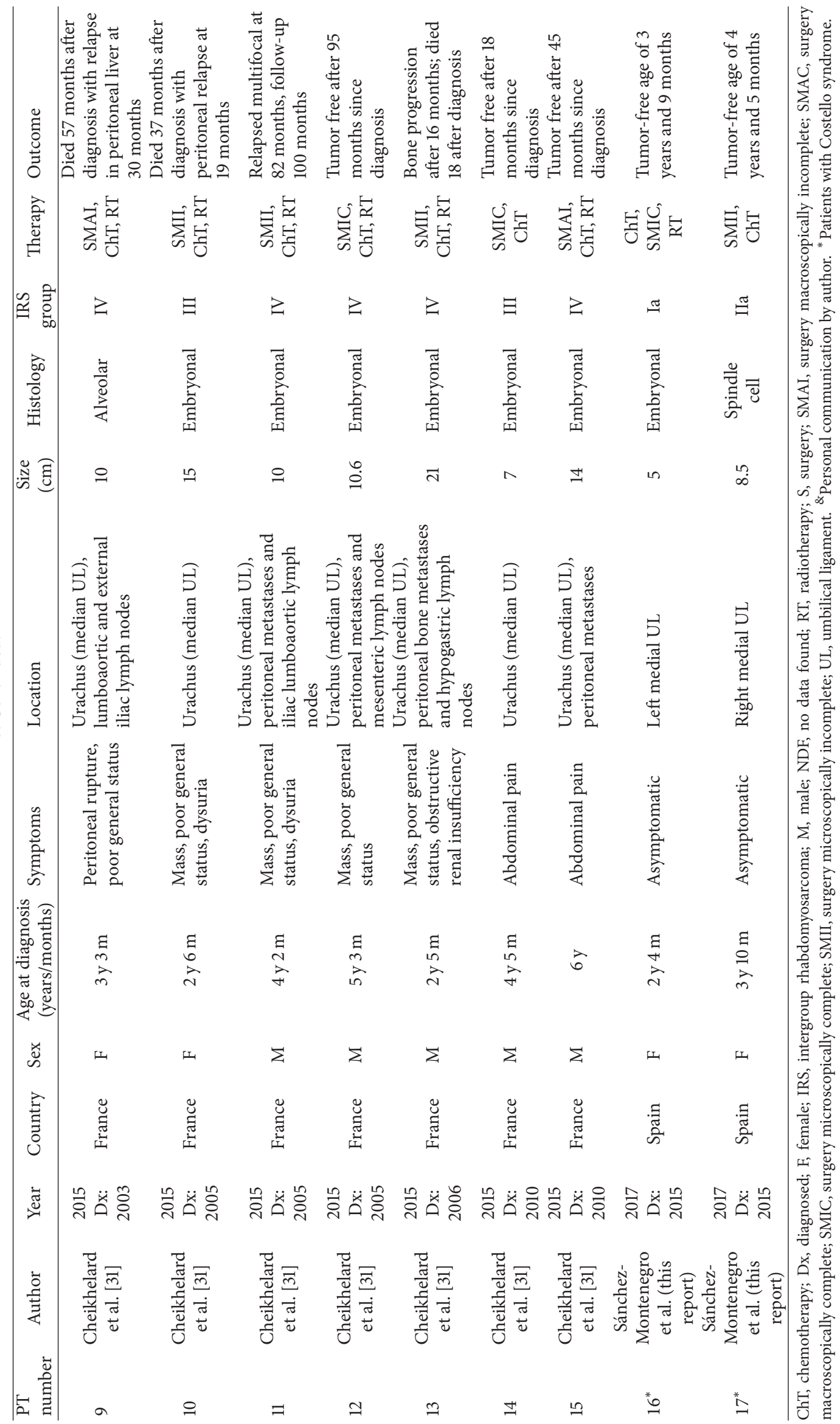


TABLE 4: Chemotherapy of pediatric patients with umbilical ligament rhabdomyosarcoma.

\begin{tabular}{|c|c|}
\hline $\begin{array}{l}\text { PT } \\
\text { number }\end{array}$ & Chemotherapy protocol \\
\hline $1-2$ & No ChT $[26,28]$ \\
\hline 3 & After establishment of the final diagnosis, he was treated with combined ChT, including VCR, ACD, and CTX [29] \\
\hline $4^{*}$ & $\begin{array}{l}\text { Initially IFOS, ACD, and VCR. After } 4 \text { courses, CTX was substituted for IFOS because of hemorrhagic cystitis. Tx complicated } \\
\text { by recurrent episodes of moderately severe diarrhoea and febrile neutropenia requiring readmission to hospital. Tx was } \\
\text { completed after six courses of ChT [7] }\end{array}$ \\
\hline 5 & NDF [30] \\
\hline 6 & $\begin{array}{l}\text { PT started induction of ChT } 2 \text { weeks after S. Induction regimen included } 9 \text { cycles of IVA (IFOS, VCR, and ACD) that were well } \\
\text { tolerated with minimal systemic complications (Tx } 953 \text { of the MMT } 95 \text { SIOP protocol for RMS) [27] }\end{array}$ \\
\hline $7^{*}$ & ChT according to the cooperative soft tissue sarcoma protocol (CWS-2002 P) was well tolerated by the PT [24] \\
\hline 8 & Adjuvant ChT with 10 cycles of IFOS, VCR, and ACD [31] \\
\hline 9 & Adjuvant ChT with 6 cycles of IFOS, VCR, and VP-16 and 9 cycles of VCR, ACD, CTX/ACD, and CTX [31] \\
\hline 10 & Neoadjuvant ChT with 6 cycles of IFOS, VCR, and ACD and adjuvant ChT with 3 cycles of IFOS, VCR, and ACD [31] \\
\hline 11 & $\begin{array}{l}\text { Neoadjuvant ChT with } 1 \text { cycle of CTX, VCR, and PRED, } 2 \text { cycles IFOS, VCR, and ACD, and } 4 \text { cycles of IFOS, VCR, ACD, and } \\
\text { DOX and adjuvant ChT with } 3 \text { cycles of IFOS, VCR, and ACD [31] }\end{array}$ \\
\hline 12 & $\begin{array}{l}\text { Neoadjuvant ChT with } 1 \text { cycle of CTX, VCR, and PRED and } 5 \text { cycles of IFOS, VCR, ACD, and DOX and adjuvant ChT with } 2 \\
\text { cycles of IFOS, VCR, ACD, and DOX and } 1 \text { cycle of IFOS, VCR, and ACD [31] }\end{array}$ \\
\hline 13 & $\begin{array}{l}\text { Neoadjuvant ChT with } 2 \text { cycles of VCR, ACD, and CTX + and } 3 \text { cycles of IFOS, VCR, ACD, and DOX and adjuvant ChT with } 3 \\
\text { cycles of IFOS, VCR, and ACD [31] }\end{array}$ \\
\hline 14 & Neoadjuvant ChT with 4 cycles of IFOS, VCR, ACD, and DOX and adjuvant ChT with 5 cycles of IFOS, VCR, ACD [31] \\
\hline 15 & $\begin{array}{l}\text { Neoadjuvant ChT with } 4 \text { cycles of IFOS, VCR, ACD, DOX, } 3 \text { cycles of IFOS, VCR, ACD and adjuvant ChT with } 1 \text { cycle of IFOS, } \\
\text { VCR, ACD, } 1 \text { cycle of IFOS, VCR [31] }\end{array}$ \\
\hline $16^{*}$ & $\begin{array}{l}\text { ChT in the standard risk group protocol EpSSG RMS } 2005 \text { subgroup D, } 3 \text { preoperative cycles of IFOS ( } 2 \text { doses at } 100 \mathrm{mg} / \mathrm{kg}) \text {, } \\
\text { VCR }(0.05 \mathrm{mg} / \mathrm{kg}) \text { and ACD }(0.05 \mathrm{mg} / \mathrm{kg})\end{array}$ \\
\hline $17^{*}$ & $\begin{array}{l}\text { ChT (postoperative } 15 \text { days) in the standard risk group protocol EpSSG RMS } 2005 \text { subgroup B (Stage I), cycles of IFOS (2 doses } \\
\left.\text { of } 3 \mathrm{~g} / \mathrm{m}^{2} / \mathrm{d}\right), \operatorname{VCR}\left(1.5 \mathrm{mg} / \mathrm{m}^{2}\right) \text { and ACD }\left(1.5 \mathrm{mg} / \mathrm{m}^{2}\right) \text { was initiated }\end{array}$ \\
\hline
\end{tabular}

ACD, actinomycin D; ChT, chemotherapy; CTX, cyclophosphamide; CWS, Cooperative Weichteilsarkom Studie (Cooperative Soft Tissue Sarcoma Study); DOX, Doxorubicin; EpSSG, European pediatric Soft tissue sarcoma Study Group; IFOS, ifosfamide; MMT, malignant mesenchymal tumor; NDF, no data found; PRED, prednisone; PT, patient; RMS, rhabdomyosarcoma; S, surgery; SIOP, Société Internationale D’Oncologie Pédiatrique (International Society of Pediatric Oncology); Tx, treatment; VCR, vincristine; VP-16, etoposide. * Patients with Costello syndrome.

that alveolar RMS and embryonal RMS are two different disorders: while alveolar RMS may originate from primitive uncommitted mesodermal cells, embryonal RMS originates probably from more differentiated myoblasts. Ras proteinspecific guanine nucleotide-releasing factor 1 (RasGRF1) plays an important role in alveolar RMS pathogenesis. These interesting concepts however need more evidence [33].

The umbilicus caudally originates the common ligament, which is divided into obliterated umbilical arteries (medial $\mathrm{UL}$ ) and urachus (median UL). The urachus varies from 3 to $10 \mathrm{~cm}$ in length and from 8 to $10 \mathrm{~mm}$ in diameter. It is a three-layered tubular structure, the innermost layer being lined with transitional epithelium in $70 \%$ of cases and with columnar epithelium in $30 \%$. The structure is surrounded by connective tissue and outermost muscular layer in continuity with the detrusor muscle. Along its path from the bladder to the umbilicus, the urachus lies between the transverse fascia and the parietal peritoneum contained in the pyramidal, retropubic, perivesical preperitoneal space compartmentalized by umbilicovesical fascia, along with the medial umbilical ligaments and the bladder. Occasionally, the urachus may merge with one or both of the obliterated umbilical arteries (medial UL), and there may be a slight deviation to the right or left of the midline [34]. The urachus is an embryological remnant resulting from the obliteration of the allantoic channel, which attaches the bladder dome to the umbilicus [31].

Urachal malignant tumors are extremely rare, representing less than $0.5 \%$ of all bladder cancers and $0.01 \%$ of all tumors. They may arise from any portion of the urachus and are most commonly found in adults and males (60-70\%). Although sarcomas represent only $8 \%$, they are the most frequent urachal neoplasms reported in patients younger than 20 years of age (67\%) [27]. Poor prognosis is a common feature in adults and pediatric urachal malignancies with $50 \%$ to $60 \%$ of 5 -year survival. In contrast, the 5 -year event-free survival in pelvic RMS was reported to be about 51\% [31].

This indolent and rather large urachal tumor was mostly revealed by an uncomplicated progressing mass without prodromic urological symptoms. The delay of diagnosis could be explained by a long asymptomatic progression of the tumor in a preperitoneal location, which allows an important local spreading before diagnosis. Even in patients without tumor rupture, peritoneal involvement appeared to be the key point in the prognosis of urachal RMS. Systematic cytology at diagnosis could be more accurate to evaluate peritoneal 
extension in order to avoid understaging and adjust adjuvant locoregional treatment [31].

Previously, only 12 patients with RMS arising from the urachus (median UL) in children without CS have been reported in the English literature (Table 3). By 2011, there were 29 reported cases of cancer in patients with CS: 19 RMS (mean age of 2.3 years; 9 embryonal, 1 alveolar, 1 mixed histology, 1 pleomorphic, 1 spindle cell type, and 6 unclassified), 5 neuroblastomas, 4 bladder cancer, and 1 fibrosarcoma [35].

Loss of heterozygosity for 11p15.5 in RMS from individuals with CS suggests that loss of the wild-type allele is the second hit in tumor development $[6,16,36]$. Whereas the HRAS mutation in a sporadic malignancy in the general population is present only within the tumor cells, in CS an identical mutation is present in every single cell of the individual, thus accounting for the tumor predisposition [1], implicating an alternate mechanism of rhabdomyosarcogenesis [16]. In addition, one patient with CS and uniparental trisomy due to triplication of the mutated paternal HRAS gene was also reported. RMS typically displays loss of heterozygosity of the 11p15.5 region because of paternal uniparental disomy, and approximately $28 \%$ of cases harbor mutations in genes of growth signaling pathways. In reports of uniparental disomy plus HRAS mutation, the latter was heterozygous and therefore must have occurred after the development of uniparental disomy [24]. The functional analysis also revealed that CS-causing mutant HRAS proteins caused oncogene-induced senescence in human fibroblasts [37]. The important role of PI3K-protein-kinase B (AKT) signaling in Ras-mediated transformation and tumorigenesis is highlighted by constitutive AKT activation in RMS cell lines and clinical samples. Thus, prolonged PI3K-AKT signaling might also be implicated in the development of soft tissue sarcoma in patients with CS [38].

Only four out of 11 patients were diagnosed with CS prior to the RMS diagnosis, in 5 patients CS was diagnosed after the RMS, and in the remaining 2 the order was unknown [20]. In 2003, a further three unpublished cases of RMS in children with CS were known through the International CS Support Group [18], and for that reason, there is possibly underdiagnosis of RMS in patients with CS.

A screening protocol was proposed consisting of ultrasound examination of the abdomen and pelvis every 36 months until age of 8-10 years for RMS and abdominal neuroblastoma, urine catecholamine metabolite analysis every 6-12 months until age of 5 years for neuroblastoma, and urinalysis for hematuria annually for bladder carcinoma after age of 10 years. The prior diagnosis of CS was the prerequisite for the implementation of a tumor screening protocol [8]. The recommendation based on the presumed fast doubling time of RMS was abdominal sonography screening for RMS that should be done in similar intervals of every four months until stronger evidence would suggest otherwise [39]. Screening for neuroblastoma is no longer recommended because many CS patients without identifiable tumor show elevated catecholamine metabolites in urine; it appears that in this patient group an elevation above the normal limit, defined as 2 standard deviations (SD) above the mean for age, is more likely to be a variant, rather than a sign of a neuroblastoma [1, 40]. A French monitoring protocol proposed the determination of catecholamines and their metabolites in urine every six months until the age of 5 years and ultrasound abdominopelvic every six months until the end of the puberty, seeking hematuria dipstick from 6 months to the age of 10 [41]. The protocol in our hospital for patients with CS includes an abdominal and pelvic ultrasound 3 times during the first year of life and in childhood every 6 months until age of 10 [42]. We believe that the best option is to perform a pelvic abdominal ultrasound every 3 months during the first eight years of life and then controls according to medical criteria. This is even more necessary in patients with possible UL RMS because diagnosis was usually done in advanced stages with a poor survival. It is possible that with close monitoring after an early diagnosis will detect initial stages of the tumor in previously not described locations.

Head and neck MRI scans can be performed less frequently. Ear examination and tympanometry every 4 to 6 months to detect middle ear effusion, which would raise suspicion for a nasopharyngeal mass, are also recommended [6].

The outcome of CS patients with RMS has been comparable to that for patients without the syndrome, with a 5-year survival rate of $65 \%$ to $70 \%$ [19]; but according to this review it could be worse than the general population. Neoplasia was noted at the cause of death in five (22\%) patients with CS $[4,15]$, but in our study the mortality associated with RMS was $43 \%$. Because the long-term survival in RMS is based on the histology of the tumor and the extent of disease at diagnosis, early diagnosis may alter the treatment regimen and improve prognosis. This could be particularly important in patients with CS, who often are medically fragile [20].

The Ras/MAPK pathway is an attractive target in the treatment of cancer utilizing small molecule therapeutics that specifically inhibits the pathway. Ras pathway agents, such as farnesyltransferase inhibitors (tipifarnib and lonafarnib) that prevent posttranslational modification of Ras, are being evaluated for cancer treatment and may be of therapeutic use for syndromes in this pathway, especially CS. In addition, B-Raf protooncogene serine/threonine kinase (BRAF) and mitogen/extracellular signal-regulated kinase (MEK) inhibitors offer the same potential in the possible treatment of CS. Since the Ras/MAPK pathway has targets for inhibition in cancer treatment, there are many small molecule therapeutics that are in development or undergoing clinical trials, with some already FDA approval [43].

\section{Conclusions}

CS is a syndrome poorly known in oncology, but their predisposition to malignancies including UL RMS implies the need for a new perspective on early diagnosis and aggressive medical and surgical treatment.

\section{Consent}

Informed consent was obtained from the new two individual participants included in the study and has been properly documented with the parental approval to the use of patient's images and complementary studies. 


\section{Competing Interests}

The authors declare that there is no conflict of interests regarding the publication of this paper.

\section{Acknowledgments}

Special thanks are due to Israel Fernández Pineda, M.D., Assistant Member and Surgery Fellowship Program Director, St. Jude Children's Research Hospital (Memphis, TN), for the recommendations and corrections to the manuscript.

\section{References}

[1] E. Quezada and K. W. Gripp, "Costello syndrome and related disorders," Current Opinion in Pediatrics, vol. 19, no. 6, pp. 636644, 2007.

[2] J. M. Costello, "A new syndrome," New Zealand Medical Journal, vol. 74, article 397, 1971.

[3] J. M. Costello, "A new syndrome: mental subnormality and nasal papillomata," Australian Paediatric Journal, vol. 13, no. 2, pp. 114-118, 1977.

[4] K. W. Gripp and A. E. Lin, "Costello syndrome: a Ras/mitogen activated protein kinase pathway syndrome (rasopathy) resulting from HRAS germline mutations," Genetics in Medicine, vol. 14, no. 3, pp. 285-292, 2012.

[5] B. Kerr, M.-A. Delrue, S. Sigaudy et al., "Genotype-phenotype correlation in Costello syndrome: HRAS mutation analysis in 43 cases," Journal of Medical Genetics, vol. 43, no. 5, pp. 401-405, 2006.

[6] N. Ahmadi and E. Harley, "Costello syndrome and the importance of cancer screening," Archives of Otolaryngology-Head and Neck Surgery, vol. 136, no. 10, pp. 1028-1029, 2010.

[7] B. Kerr, O. B. Eden, R. Dandamudi et al., "Costello syndrome: two cases with embryonal rhabdomyosarcoma," Journal of Medical Genetics, vol. 35, no. 12, pp. 1036-1039, 1998.

[8] K. W. Gripp, C. I. Scott Jr., L. Nicholson et al., "Five additional Costello syndrome patients with rhabdomyosarcoma: proposal for a tumor screening protocol," American Journal of Medical Genetics, vol. 108, no. 1, pp. 80-87, 2002.

[9] K. W. Gripp, A. E. Lin, D. L. Stabley et al., "HRAS mutation analysis in Costello syndrome: genotype and phenotype correlation," American Journal of Medical Genetics Part A, vol. 140, no. 1, pp. 1-7, 2006.

[10] M. Feingold, "Costello syndrome and rhabdomyosarcoma," Journal of Medical Genetics, vol. 36, no. 7, pp. 582-583, 1999.

[11] S. M. White, J. M. Graham Jr., B. Kerr et al., "The adult phenotype in Costello syndrome," American Journal of Medical Genetics A, vol. 136, no. 2, pp. 128-135, 2005.

[12] G. Bisogno, A. Murgia, I. Mammi, M. S. Strafella, and M. Carli, "Rhabdomyosarcoma in a patient with cardio-facio-cutaneous syndrome," Journal of Pediatric Hematology/Oncology, vol. 21, no. 5, pp. 424-427, 1999.

[13] S. Sigaudy, G. Vittu, A. David et al., "Costello syndrome: report of six patients including one with an embryonal rhabdomyosarcoma," European Journal of Pediatrics, vol. 159, no. 3, pp. 139-142, 2000.

[14] A. Hinek, M. A. Teitell, L. Schoyer et al., "Myocardial storage of chondroitin sulfate-containing moieties in Costello syndrome patients with severe hypertrophic cardiomyopathy," American Journal of Medical Genetics, vol. 133, no. 1, pp. 1-12, 2005.
[15] A. E. Lin, P. D. Grossfeld, R. M. Hamilton et al., "Further delineation of cardiac abnormalities in Costello syndrome," American Journal of Medical Genetics, vol. 111, no. 2, pp. 115-129, 2002.

[16] A. L. Estep, W. E. Tidyman, M. A. Teitell, P. D. Cotter, and K. A. Rauen, "HRAS mutations in Costello syndrome: detection of constitutional activating mutations in codon 12 and 13 and loss of wild-type allele in malignancy," American Journal of Medical Genetics, vol. 140, no. 1, pp. 8-16, 2006.

[17] H. Kawame, M. Matsui, K. Kurosawa et al., "Further delineation of the behavioral and neurologic features in Costello syndrome," American Journal of Medical Genetics, vol. 118A, no. 1, pp. 8-14, 2003.

[18] B. Kerr, M. A. Einaudi, P. Clayton et al., "Is growth hormone treatment beneficial or harmful in Costello syndrome?" Journal of medical genetics, vol. 40, no. 6, article e74, 2003.

[19] J. P. O’Neal, J. Ramdas, W. E. Wood, and P. K. Pellitteri, "Parameningeal rhabdomyosarcoma in a patient with Costello syndrome," Journal of Pediatric Hematology/Oncology, vol. 26, no. 6, pp. 389-392, 2004.

[20] K. W. Gripp, “Tumor predisposition in Costello syndrome," American Journal of Medical Genetics Part C: Seminars in Medical Genetics, vol. 137C, no. 1, pp. 72-77, 2005.

[21] Y. Aoki, T. Niihori, H. Kawame et al., "Germline mutations in HRAS proto-oncogene cause Costello syndrome," Nature Genetics, vol. 37, no. 10, pp. 1038-1040, 2005.

[22] Y. Abe, Y. Aoki, S. Kuriyama et al., "Prevalence and clinical features of Costello syndrome and cardio-facio-cutaneous syndrome in Japan: findings from a nationwide epidemiological survey," American Journal of Medical Genetics Part A, vol. 158, no. 5, pp. 1083-1094, 2012.

[23] I. F. M. Lo, C. Brewer, N. Shannon et al., "Severe neonatal manifestations of Costello syndrome," Journal of Medical Genetics, vol. 45, no. 3, pp. 167-171, 2008.

[24] J. Menke, S. Pauli, M. Sigler et al., "Uniparental trisomy of a mutated HRASproto-oncogene in embryonal rhabdomyosarcoma of a patient with costello syndrome," Journal of Clinical Oncology, vol. 33, no. 13, pp. e62-e65, 2015.

[25] C. P. Kratz, L. Franke, H. Peters et al., "Cancer spectrum and frequency among children with Noonan, Costello, and cardiofacio-cutaneous syndromes," British Journal of Cancer, vol. 112, no. 8, pp. 1392-1397, 2015.

[26] H. K. Ransom, "Sarcoma of the urachus: review of the literature with report of an additional case," The American Journal of Surgery, vol. 22, no. 2, pp. 187-191, 1933.

[27] E. M. L.-T. Fernández, N. H. Siverio, R. L. Almaraz, L. M. Viota, J. R. Luis, and L. D. Flores, "Radical surgery and IVAchemotherapeutic regimen to treat embryonal rhabdomyosarcoma of the urachus: case report," Pediatric Hematology and Oncology, vol. 24, no. 7, pp. 543-550, 2007.

[28] M. Ishikawa, T. Nishi, R. Yamada et al., "A case of urachal sarcoma in childhood: case report and review of the world literature," Journal of the Japanese Society of Pediatric Surgeons, vol. 21, pp. 128-135, 1985.

[29] S. Yokoyama, Y. Hayashida, J. Nagahama et al., "Rhabdomyosarcoma of the urachus: a case report," Acta Cytologica, vol. 41, no. 4, pp. 1293-1298, 1997.

[30] U. Schulz and C. P. O'Leary, "Spinal AVM, epidermal nevus, and rhabdomyosarcoma: a rare neurocutaneous syndrome?" Neurology, vol. 56, no. 3, pp. 395-397, 2001. 
[31] A. Cheikhelard, S. Irtan, D. Orbach et al., "Urachal rhabdomyosarcoma in childhood: a rare entity with a poor outcome," Journal of Pediatric Surgery, vol. 50, no. 8, pp. 1329-1333, 2015.

[32] K. W. Gripp, E. Hopkins, K. Sol-Church et al., "Phenotypic analysis of individuals with Costello syndrome due to HRAS p.G13C," American Journal of Medical Genetics, Part A, vol. 155, no. 4, pp. 706-716, 2011.

[33] M. Tarnowski, G. Schneider, G. Amann et al., "RasGRF1 regulates proliferation and metastatic behavior of human alveolar rhabdomyosarcomas," International Journal of Oncology, vol. 41, no. 3, pp. 995-1004, 2012.

[34] J.-S. Yu, K. W. Kim, H.-J. Lee, Y.-J. Lee, C.-S. Yoon, and M.J. Kim, "Urachal remnant diseases: spectrum of CT and US findings," Radiographics, vol. 21, no. 2, pp. 451-461, 2001.

[35] C. P. Kratz, S. Rapisuwon, H. Reed, H. Hasle, and P. S. Rosenberg, "Cancer in Noonan, Costello, cardiofaciocutaneous and LEOPARD syndromes," American Journal of Medical Genetics, Part C: Seminars in Medical Genetics, vol. 157, no. 2, pp. 83-89, 2011.

[36] B. Kerr, M. L. Mucchielli, S. Sigaudy et al., "Is the locus for Costello syndrome on 11p?" Journal of Medical Genetics, vol. 40, no. 6, pp. 469-471, 2003.

[37] T. Niihori, Y. Aoki, N. Okamoto et al., "HRAS mutants identified in Costello syndrome patients can induce cellular senescence: possible implications for the pathogenesis of Costello syndrome," Journal of Human Genetics, vol. 56, no. 10, pp. 707-715, 2011.

[38] G. Rosenberger, S. Meien, and K. Kutsche, “Oncogenic HRAS mutations cause prolonged PI3K signaling in response to epidermal growth factor in fibroblasts of patients with costello syndrome," Human Mutation, vol. 30, no. 3, pp. 352-362, 2009.

[39] M. R. DeBaun, "Screening for cancer in children with Costello syndrome," American Journal of Medical Genetics, vol. 108, no. 1, pp. 88-90, 2002.

[40] K. W. Gripp, H. Kawame, D. H. Viskochil, and L. Nicholson, "Elevated catecholamine metabolites in patients with Costello syndrome," American Journal of Medical Genetics, vol. 128, no. 1, pp. 48-51, 2004.

[41] M.-A. Delrue, B. Arveiler, and D. Lacombe, "Costello syndrome: clinical features and tumoral risk," Archives de Pediatrie, vol. 9, no. 10, pp. 1059-1063, 2002.

[42] V. Martínez-González and P. Lapunzina, Síndrome de Costello: Grupo de Trabajo sobre Cáncer en Síndrome Genéticos Polimarformativos (GT-CSGP), https://www.orpha.net/data/patho/Pro/ es/CSGP-Costello-3.pdf.

[43] K. A. Rauen, A. Banerjee, W. R. Bishop et al., "Costello and cardio-facio-cutaneous syndromes: moving toward clinical trials in RASopathies," American Journal of Medical Genetics Part C: Seminars in Medical Genetics, vol. 157, no. 2, pp. 136-146, 2011. 


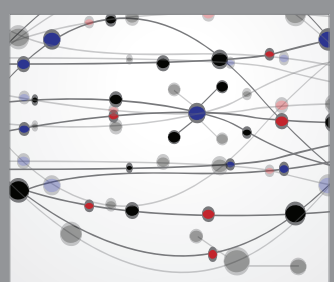

The Scientific World Journal
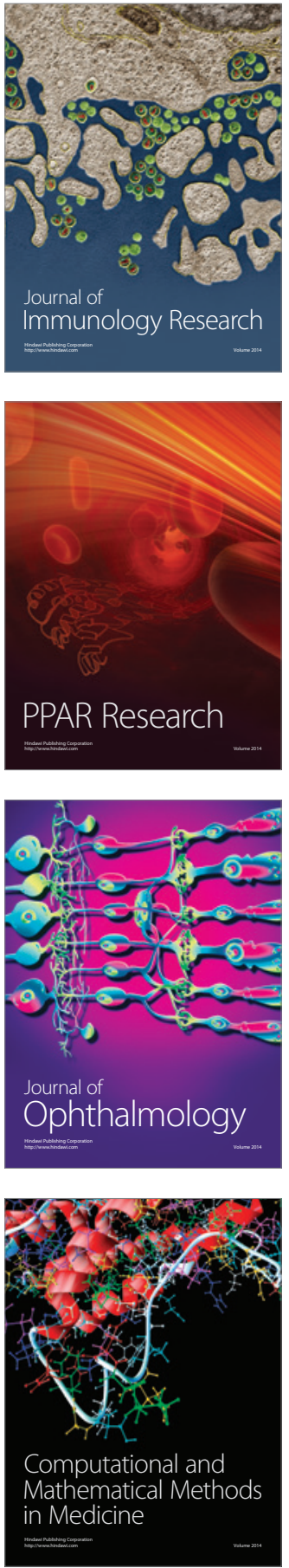

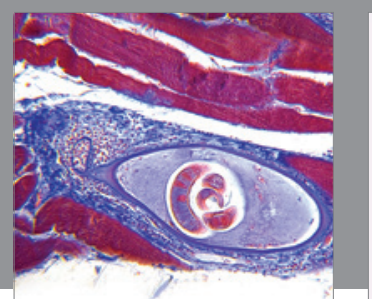

Gastroenterology Research and Practice
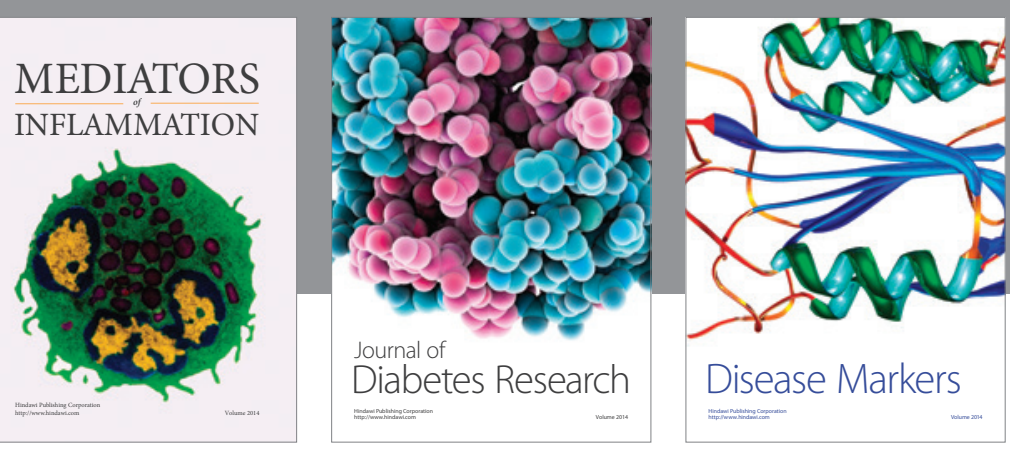

Disease Markers

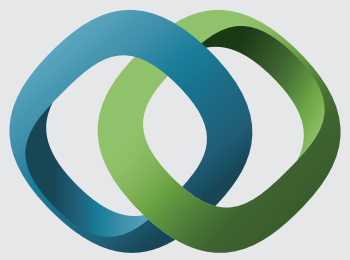

\section{Hindawi}

Submit your manuscripts at

https://www.hindawi.com
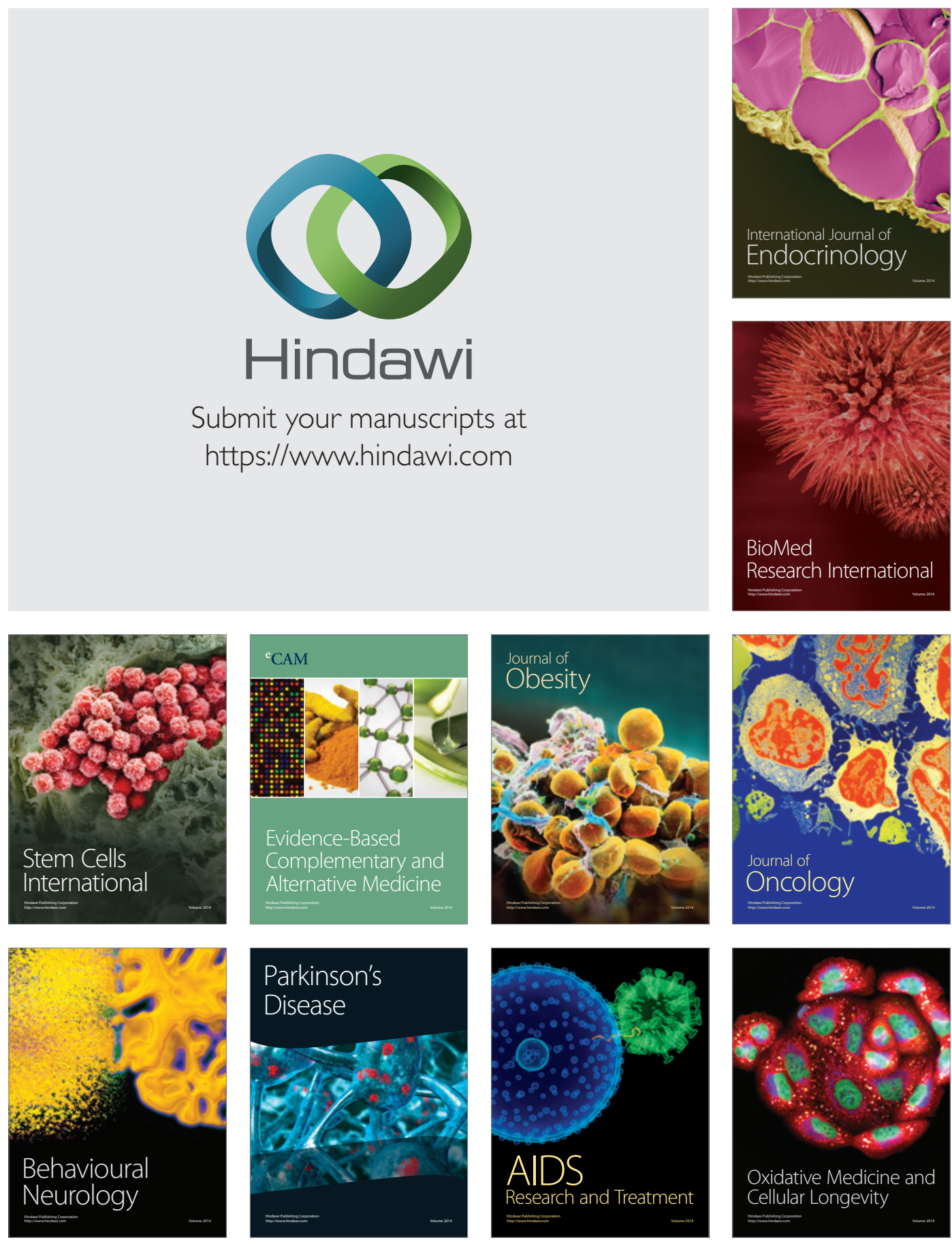